\title{
Spectroscopic analysis of two peculiar emission-line stars: RJHA 49 and SS7321
}

\author{
C. B. Pereira ${ }^{1}$, W. L. F. Marcolino ${ }^{1}$, M. Machado ${ }^{2}$, and F. X. de Araújo ${ }^{1}$ \\ 1 Observatório Nacional-MCT, Rua José Cristino 77, CEP 20921-400 São Cristóvão, Rio de Janeiro-RJ, Brazil \\ e-mail: [claudio; wagner; araujo]@on.br \\ 2 Departamento de Física, UERJ, Rua S. Francisco Xavier 400, CEP 12322-100 Rio de Janeiro-RJ, Brazil
}

Received 18 April 2007 / Accepted 15 July 2007

ABSTRACT

\begin{abstract}
Aims. We investigate the spectra and the evolutionary stages of two peculiar emission-line stars: RJHA 49 and SS73 21. Methods. We used low and high resolution optical data. Line identifications and measurements were performed for several features in their spectra.

Results. For each object, we derived the extinction and the excitation temperature from a set of [Fe II] lines and the electron density from [N II] lines. For RJHA 49, no detailed spectroscopic study has been done so far. Our low resolution spectrum have confirmed the main characteristics found in previous works on SS73 21. On the other hand, from our high resolution data, we find that the H $\alpha$ line presents a double peak, in contrast with the suggestion in the literature that it should reveal a P-Cygni profile. Surprisingly, we found a few He I transitions resembling P-Cygni profiles (e.g. He I 15876), directly suggesting that mass loss is active in SS73 21 . We also discuss the nature of these two objects based on data obtained. Although the evolutionary status of SS73 21 seems well established from previous studies (a proto-planetary nebula), the situation for RJHA 49 is not very clear mainly due to its unknown distance. However, from the strength of [N II] $\lambda 5754$ relative to [O I] $\lambda 6300$, the possibility of RJHA 49 being a LBV object is reduced, and a $\mathrm{B}[\mathrm{e}]$-supergiant or a proto-planetary nebula status is more plausible.
\end{abstract}

Key words. stars: emission-line, Be

\section{Introduction}

The spectral characteristics of a group of stars known as "B[e]-stars" (B-type stars with forbidden emission lines, mainly from iron, in the optical spectrum) have received wide attention in recent years. Interestingly, different groups of objects in distinct and well defined evolutionary stages may present a very similar spectrum with Balmer lines, several permitted and forbidden iron emissions, as well as an infrared excess. Due to this fact, it was proposed in the literature that all these objects should be categorized as stars with the " $\mathrm{B}[\mathrm{e}]-$ phenomenon" (Lamers et al. 1998). Yet, there is a group that still resists being properly identified, namely, the "unclassified B[e] stars" (or unclB[e] stars). Although some stars within this group have been studied more than others (e.g. HD 45677 and HD 50138; Lamers et al. 1998), the nature and the evolutionary status of most of them has not been revealed yet. An interesting review of the observational properties of different subgroups of $\mathrm{B}[\mathrm{e}]-$ stars and a comparison to other peculiar emission-line objects, including the unclB $[\mathrm{e}]$ class, has been recently presented by Miroshnichenko (2006).

Following our program at the European Southern Observatory (ESO) investigating those emission-line objects in the southern hemisphere, we present spectroscopic data

\footnotetext{
* Based on observations made with the $1.52 \mathrm{~m}$ telescope at the European Southern Observatory (La Silla, Chile) under the agreement with the Observatório Nacional, Brazil.

$\star \star$ Spectra and fits files are only available in electronic form at the CDS via anonymous ftp to cdsarc.u-strasbg.fr (130.79.128.5) or via
}

http://cdsweb.u-strasbg.fr/cgi-bin/qcat?J/A+A/477/877 in this paper for two peculiar emission-line stars: RJHA 49 (=MWC 819) and SS73 21 (=Th 35-27). Both objects were selected from the works of Sanduleak \& Stephenson (1973) and Allen \& Swings (1976). Previous efforts of our program led to classification of 33 emission-line stars (Pereira et al. 2003a) and analysis of three stars with $\eta$-Car spectrum: SS73 11 (Landaberry et al. 2001), SS73 56, and Hen 2-79 (Pereira et al. 2003b).

There are not many objects that have a $\eta$-Car type spectrum. In the early 70's, Swings \& Allen (1973) realized that the spectral characteristics in the visual of MWC 645 and MWC 819 (=RJHA 49) looked very similar to $\eta$-Car. MWC 645 has later investigated by Jaschek et al. (1996). RJHA 49 has already been classified as a possible planetary nebula (Kohoutek 1971), a Be star with infrared excess (Allen \& Swings 1976) and more recently, as a $\mathrm{B}[\mathrm{e}]$ star by The et al. (1994) and an unclB[e] by Lamers et al. (1998). According to Miroshnichenko (2006), RJHA 49 is one of the objects that has "received almost no attention since the introduction of B[e] stars". On the other hand, the nature of SS7321 seems to be better established. On the basis of infrared IRAS colors, Parthasarathy \& Pottasch (1989) first suggested that this object could be a proto-planetary nebula. Indeed, different recent studies based on images and lowresolution spectroscopy have supported this view (García-Lario et al. 1999; Sahai et al. 1999; Parthasarathy et al. 2001). In the present paper, the high- resolution optical spectrum of SS73 21 is being investigated for the first time.

The rest of the paper is divided in the following manner: in Sect. 2 we present the details of our observational data, including the reduction procedure, and the extinction derived for each object. In Sect. 3 we present some physical conditions derived 
for both objects: the excitation temperature from forbidden iron lines and the electron density from forbidden nitrogen lines. In Sect. 4 we discuss their nature, and finally in the last section we summarize the main points of our work.

\section{The data}

\subsection{Observations and reduction}

The low-resolution spectroscopic observations were performed using a Boller \& Chivens spectrograph at the Cassegrain focus of the ESO 1.52 m telescope in La Silla (Chile) on February 4, 1999 (RJHA 49) and March 2, 1999 (SS73 21). A UV-flooded, thinned Loral Lesser CCD \#39 (2048 × 2048, $15 \mu \mathrm{m} /$ pixel) was used as the detector, because it gives high quantum efficiency in the blue and in the UV. We used the grating \#23 with $600 \mathrm{l} / \mathrm{mm}$, providing a resolution of about $4.6 \AA$ in the range $\sim 4000-8000 \AA$. The slit orientation was east-west and the slit width was $2^{\prime \prime}$. The sky conditions in these observations were mostly clear, but not photometric with a mean seeing of $1.5^{\prime \prime}$, therefore the flux calibration should be viewed with caution.

The spectra were reduced using standard IRAF tasks, from bias subtraction and flat-field correction, through spectral extraction and wavelength and flux calibration. Spectrophotometric standards from Oke (1974) and Hamuy et al. (1994) were observed.

In the linearized spectra, the fluxes of emission lines were measured by the conventional method adjusting a Gaussian function to the line profile, thereby obtaining the intensity, the central wavelength, and the line width at half power level. Uncertainties in the line intensities come mainly from the position of the underlying continuum. We estimate the errors in the fluxes to be about $20 \%$ for weaker lines (line fluxes about 10 on the scale of $\mathrm{H} \beta=100$ ) and about $10 \%$ for stronger lines.

RJHA 49 and SS73 21 were also observed in high-resolution mode with FEROS in the $1.52 \mathrm{~m}$ ESO telescope in La Silla (Chile) on February 9 and February 3 2001, respectively. The FEROS spectral resolving power is $R=48000$, corresponding to 2.2 pixels of $15 \mu \mathrm{m}$. The total wavelength coverage is 4000-9200 $\AA$, and the nominal S/N measured by the rms flux fluctuation is approximately 100 after $3600 \mathrm{~s}$ of exposure time. The spectra were reduced with the MIDAS pipeline reduction (Kaufer et al. 1999) package consisting of the following standard steps: CCD bias correction, flat-fielding, spectrum extraction, wavelength calibration, correction of barycentric velocity, and spectrum rectification.

\subsection{The spectra of RJHA 49 and SS73 21}

\subsubsection{Low resolution}

Our optical spectra for RJHA 49 and SS73 21 are displayed in Fig. 1. For comparison, we also show spectra in this figure of other objects previously analyzed by us, namely, Hen 2-79, SS73 11, and SS73 56, as well as the spectrum of $\eta$ Car, obtained at the same resolution.

The spectrum of RJHA 49 presents several strong emission lines mostly due to single ionized forbidden and permitted iron over a flat continuum. The majority of the iron features are also present in the spectrum of the objects mentioned above. The nitrogen forbidden line at $5754 \AA$ is present but weaker than the oxygen forbidden line at $6300 \AA$. The intensities of these two transitions are particularly important, since they can be used as a criterion for distinguishing for example, a $\mathrm{B}[\mathrm{e}] \mathrm{sg}$ from an
LBV star (Zickgraf 1989). As in the other stars, $\mathrm{H} \alpha$ and $\mathrm{H} \beta$ are among the strongest lines in the spectrum.

Gárcia-Lario et al. (1999) have studied the low-resolution spectrum of SS73 21 from 3500-11200 A. According to these authors, a rich emission-line spectrum can be seen, which is characterized by strong and broad emission of $\mathrm{H}$ I Balmer lines. Permitted emission lines of He I, Fe II, O I, and Ca II, as well as forbidden transitions from ions such as [Fe II], [N II], [O I], [S II], and [Ca II] are also present. These characteristics are confirmed by our more recent data. Furthermore, we find no significant line variations compared to their study. Nevertheless, as seen later in the paper, the analysis of the high-resolution spectrum of SS73 21 provided valuable additional information compared to the low-resolution data.

Overall, we can see from Fig. 1 that the spectra of RJHA 49 and SS73 21 are similar to the spectra of the objects Hen 2-79, SS73 11, SS73 56, and $\eta$ Car. The main characteristics in common are clearly the presence of several Fe II and [Fe II] emissions and the HI Balmer lines. Interestingly, with the exception of $\eta$ Car, the nature of all these peculiar stars has not yet been firmly established. For SS73 11, for example, although Landaberry et al. (2001) could conclude that this object is not a B[e]sg, a HerbigAeB[e] or a symbiotic star, these same authors argue that an LBV or a proto-planetary nebula classification is possible. For Hen 2-79 and SS73 56, a detailed analysis of their spectra favors a proto-planetary nebula status, but an evolved massive star classification cannot be discarded (Pereira et al. 2003b). Undoubtedly, one of the main difficulties in determining the nature of these and other similar objects is their unknown distance. This point and the status of RJHA 49 and SS73 21 will be discussed in Sect. 4.

In Table 1 we provide the line identification, as well as the line fluxes of transitions other than from Fe II. As can be seen, some features in the spectrum of SS73 21 are not present in RJHA 49, such as the He I and the [S II] lines. For RJHA 49, there are no previous line measurements in the literature. In addition to Table 1, the 4th and 7th columns of Table 2 list the line flux in units of $\mathrm{H} \beta=100$ of some multiplets of single ionized forbidden iron used for reddening and excitation temperature determination (see Sects. 2.3 and 3.1). For line identifications, we used the same as procedure described in Landaberry et al. (2001).

\subsubsection{High resolution}

The high-resolution spectra were used to better identify some features that are blended or were not resolved in the lowresolution spectra. This procedure allowed us to estimate the different contributions of each transition and to discover important line profiles (e.g. H $\alpha$; see below).

In Fig. 2 we show three spectral regions to better illustrate the differences in strength of some emission lines among the objects studied here (RJHA 49 and SS73 21) to those already studied in Landaberry et al. (2001) and Pereira et al. (2003b). It can be seen that the strength of the forbidden line [O I] $\lambda 6300$ is stronger than [N II] 15754 in RJHA 49, SS73 21, SS73 56, and Hen 2-79, while the opposite occurs in SS73 11.

Our optical high-resolution spectrum of SS73 21 reveals interesting additional information compared to previous studies. The most important one is that the $\mathrm{H} \alpha$ line clearly presents a double peak rather than the P-Cygni profile proposed by Parthasarathy et al. (2001) on the basis of low-resolution data. As can be seen in Fig. 2, the $\mathrm{H} \alpha$ double-peak profile is also present in the other stars. SS73 21 has the broadest profile. In 


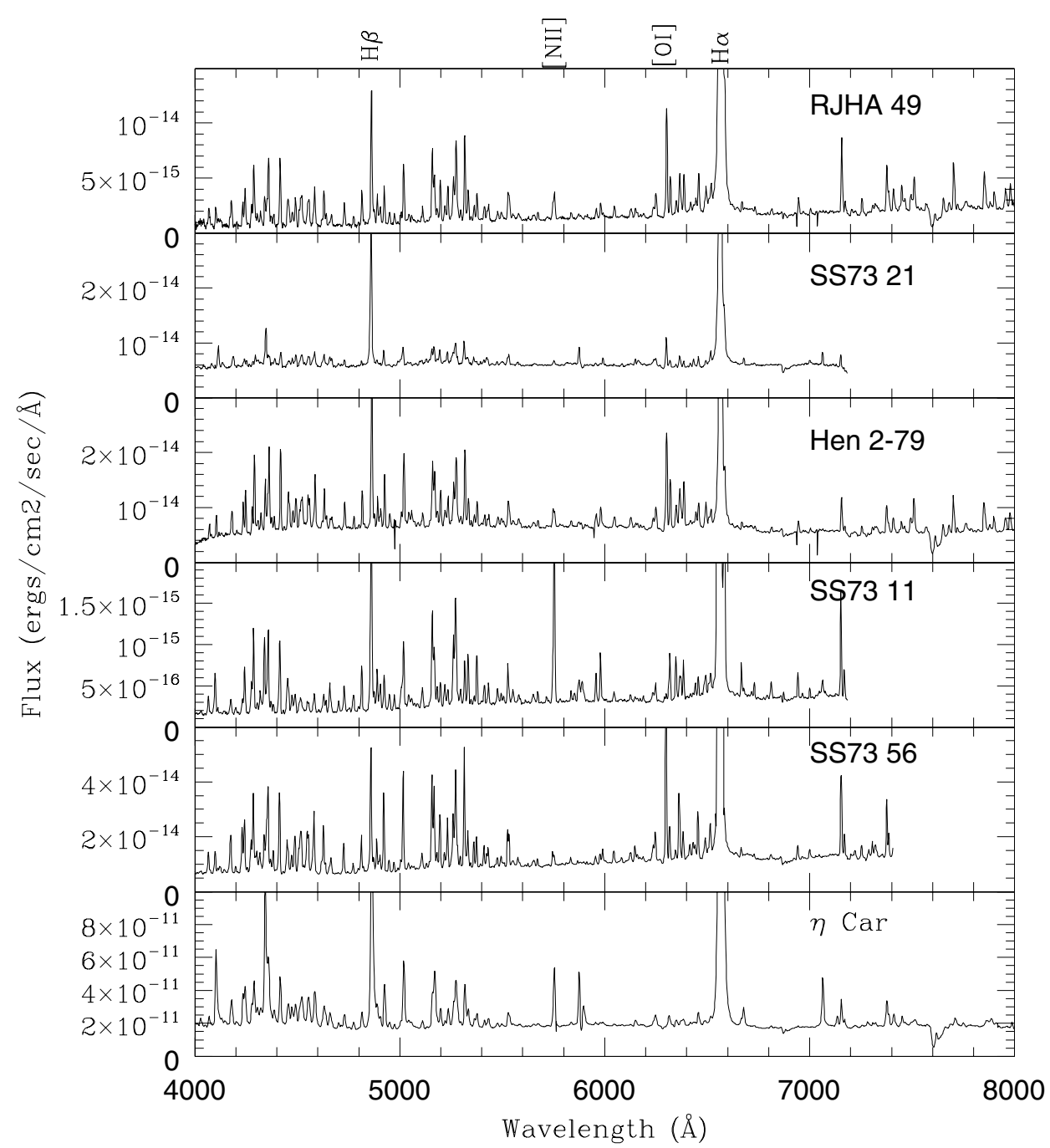

Fig. 1. Optical spectrum of RJHA 49 and SS73 21 in comparison with other peculiar emission-line stars already analyzed and with $\eta$ Car.

Table 1. Observed emission lines other than Fe II identified in the spectra of RJHA 49 and SS73 21 in units of $\mathrm{H} \beta=100.0$.

\begin{tabular}{lc|cc}
\hline \hline Wavelength & Identification & \multicolumn{2}{|c}{$F(\lambda)$} \\
& & RJHA 49 & SS73 21 \\
\hline 4101 & $\mathrm{H} \delta$ & 11.1 & 14.0 \\
4340 & $\mathrm{H} \gamma$ & 25.7 & 23.8 \\
4861 & $\mathrm{H} \beta$ & 100.0 & 100.0 \\
5754 & {$[\mathrm{~N} \mathrm{II}]$} & 18.3 & 3.5 \\
5876 & $\mathrm{He} \mathrm{I}$ & - & 10.5 \\
6300 & {$[\mathrm{O} \mathrm{I}]$} & 82.4 & 16.2 \\
6363 & {$[\mathrm{O} \mathrm{I}]$} & 33.1 & 8.6 \\
6563 & $\mathrm{H} \alpha$ & 5110.0 & 855.0 \\
6584 & {$[\mathrm{~N} \mathrm{II}]$} & 100.0 & 43.1 \\
6678 & $\mathrm{He} \mathrm{I}$ & - & 3.0 \\
6717 & {$[\mathrm{~S} \mathrm{II}]$} & - & 1.3 \\
6731 & {$[\mathrm{~S} \mathrm{II}]$} & - & 1.7 \\
7065 & $\mathrm{He} \mathrm{I}$ & - & 7.7 \\
\hline
\end{tabular}

RJHA 49, $\mathrm{H} \alpha$ has the strongest intensity, and the blue peak is more intense than the red one. Table 3 shows the intensities relative to the continuum and equivalent widths of some identified lines in the spectrum of these stars. The "blue" and "red" component mentioned in Table 3 refers to the two peaks seen in the profile of the Balmer lines.

\subsection{Extinction}

We determined the extinction parameter for RJHA 49 and SS73 21 in the same way as Pagel (1969). Our previous works on SS73 11, SS73 56, and Hen 2-79 (Landaberry et al. 2001; Pereira et al. 2003b) followed the same procedure. We first measured line fluxes of some Fe II forbidden lines (between $4100 \AA$ and $7000 \AA$ ) with an excitation potential between 2.5 and $3.2 \mathrm{eV}$. We then plot log $I$ (defined below) against the reciprocal wavelength $(1 / \lambda(\mu \mathrm{m}))$ in the abscissa. The ordinate $\log I$ is related to the difference between the logarithm of the observed flux and the logarithm of the emitted flux by the source in the same wavelength range according to

$\log I=\log \left(F_{\text {obs }}(\lambda)\right)-(\log (5000 / \lambda)+\log (g A)-0.56 \chi+2.0)$.

In the above expression, $F_{\text {obs }}(\lambda)$ is the observed line flux in units of $\mathrm{H} \beta=100, g A$ is the statistical weight multiplied by the transition probabilities, and $\chi$ is the excitation potential of the upper level of the transition. The factor 2.0 accounts for $\log F(\mathrm{H} \beta)=100$.

The 5th and 8th columns of Table 2 give $\log I$ as defined in the text for both stars. The color excess $E(B-V)$ that results from this procedure is $1.22 \pm 0.13$ for RJHA 49 and $0.77 \pm 0.07$ for SS73 21. For SS73 21, previous extinction determinations are given by García-Lario et al. (1999) and 
Table 2. Multiplets, wavelengths, excitation potential $(\chi)$, observed emission line fluxes $F(\lambda)$ in units of $\mathrm{H} \beta=100.0$, $\log I$ (defined in the text) and the parameter $\beta$ (also defined in the text) of some selected [Fe II] emission lines used for reddening and excitation temperature determination of RJHA 49 and SS73 21.

\begin{tabular}{|c|c|c|c|c|c|c|c|c|}
\hline \multirow[b]{2}{*}{ M } & \multirow[b]{2}{*}{ Identification } & \multirow[b]{2}{*}{$\chi(\mathrm{eV})$} & \multicolumn{3}{|c|}{ " RJHA 49} & \multicolumn{3}{|c|}{$\overline{\text { SS73 21 }}$} \\
\hline & & & $F(\lambda)$ & $\log I$ & $\beta$ & $F(\lambda)$ & $\log I$ & $\beta$ \\
\hline \multirow[t]{3}{*}{$4 \mathrm{~F}$} & 4639.68 & 2.77 & 5.0 & 0.23 & 4.39 & - & - & - \\
\hline & 4728.07 & 2.12 & 10.0 & -0.12 & 4.41 & - & - & - \\
\hline & 4889.63 & 2.57 & 10.0 & 0.10 & 4.38 & 2.5 & -0.50 & 3.77 \\
\hline \multirow[t]{2}{*}{$6 \mathrm{~F}$} & 4416.27 & 2.79 & 13.0 & -0.01 & 4.33 & 2.9 & -0.69 & 3.59 \\
\hline & 4457.95 & 2.82 & - & - & - & 1.4 & -0.59 & 3.58 \\
\hline \multirow[t]{5}{*}{$7 \mathrm{~F}$} & 4287.40 & 2.88 & 20.0 & 0.02 & 4.46 & 5.8 & -0.52 & 3.71 \\
\hline & 4359.34 & 2.88 & 16.0 & 0.07 & 4.38 & 4.5 & -0.48 & 3.75 \\
\hline & 4413.78 & 2.88 & 13.0 & 0.12 & 4.44 & - & - & - \\
\hline & 4452.11 & 2.88 & 9.0 & 0.17 & 4.49 & 1.7 & -0.55 & 3.68 \\
\hline & 4474.91 & 2.88 & 2.0 & -0.18 & 4.14 & - & - & - \\
\hline \multirow[t]{4}{*}{$14 \mathrm{~F}$} & 7155.14 & 1.96 & 54.0 & 0.81 & 4.71 & 8.1 & -0.01 & 4.14 \\
\hline & 7171.98 & 2.01 & - & - & - & 2.2 & -0.02 & 4.10 \\
\hline & 7388.16 & 2.02 & 9.0 & 0.72 & 4.59 & 2.2 & -0.05 & 4.24 \\
\hline & 7452.50 & 2.02 & - & - & - & 2.6 & 0.04 & 4.16 \\
\hline \multirow[t]{2}{*}{$17 \mathrm{~F}$} & 5412.64 & 2.63 & 7.0 & 0.32 & 4.50 & 1.0 & -0.53 & 3.67 \\
\hline & 5527.30 & 2.53 & 13.0 & 0.28 & 4.60 & 2.2 & -0.49 & 3.85 \\
\hline \multirow[t]{6}{*}{$18 \mathrm{~F}$} & 5107.96 & 2.77 & 3.0 & 0.36 & 4.29 & - & - & - \\
\hline & 5158.00 & 2.69 & 10.0 & 0.44 & 4.42 & 1.4 & -0.41 & 3.65 \\
\hline & 5181.97 & 2.77 & 4.0 & 0.34 & 4.27 & - & - & - \\
\hline & 5268.88 & 2.69 & 4.0 & 0.25 & 4.23 & - & - & - \\
\hline & 5273.38 & 2.57 & - & - & - & 3.6 & -0.32 & 3.80 \\
\hline & 5433.15 & 2.67 & 8.0 & 0.44 & 4.61 & - & - & - \\
\hline \multirow[t]{6}{*}{$19 \mathrm{~F}$} & 5111.63 & 2.65 & 4.0 & 0.02 & 4.09 & - & - & - \\
\hline & 5158.81 & 2.62 & 20.0 & -0.01 & 4.08 & 6.0 & -0.53 & 3.61 \\
\hline & 5220.06 & 2.66 & 4.0 & 0.07 & 4.14 & 1.2 & -0.45 & 3.69 \\
\hline & 5261.61 & 2.65 & 17.0 & 0.17 & 4.25 & 4.0 & -0.46 & 3.67 \\
\hline & 5333.65 & 2.66 & - & - & - & 2.9 & -0.43 & 3.69 \\
\hline & 5376.47 & 2.68 & 15.0 & 0.37 & 4.45 & 2.5 & -0.39 & 3.72 \\
\hline \multirow[t]{9}{*}{$20 \mathrm{~F}$} & 4814.55 & 2.79 & 9.0 & -0.10 & 3.97 & 2.0 & -0.76 & 3.34 \\
\hline & 4874.49 & 2.83 & 2.0 & -0.13 & 3.92 & - & - & - \\
\hline & 4905.35 & 2.82 & 9.0 & 0.27 & 4.33 & - & - & - \\
\hline & 4947.38 & 2.79 & 2.0 & 0.16 & 4.24 & - & - & - \\
\hline & 4950.74 & 2.84 & 4.0 & 0.36 & 4.41 & 0.5 & -0.55 & 3.52 \\
\hline & 4973.39 & 2.83 & 5.0 & 0.36 & 4.41 & 0.5 & -0.64 & 3.43 \\
\hline & 5005.52 & 2.82 & 5.0 & 0.52 & 4.58 & - & - & - \\
\hline & 5020.24 & 2.84 & 3.0 & 0.21 & 4.26 & - & - & - \\
\hline & 5043.53 & 2.83 & 5.0 & 0.70 & 4.75 & - & - & - \\
\hline \multirow[t]{8}{*}{$21 \mathrm{~F}$} & 4243.98 & 3.14 & 22.0 & 0.00 & 4.17 & 3.3 & -0.83 & 3.26 \\
\hline & 4244.81 & 3.21 & 4.0 & 0.03 & 4.16 & 0.9 & -0.62 & 3.43 \\
\hline & 4276.83 & 3.19 & 6.0 & -0.32 & 3.83 & 2.2 & -0.75 & 3.16 \\
\hline & 4319.62 & 3.21 & 4.0 & -0.29 & 3.84 & 1.4 & -0.74 & 3.30 \\
\hline & 4346.85 & 3.14 & 6.0 & 0.08 & 4.25 & 0.8 & -0.80 & 3.29 \\
\hline & 4352.78 & 3.19 & 11.0 & 0.29 & 4.42 & 1.7 & -0.47 & 3.52 \\
\hline & 4358.37 & 3.22 & 11.0 & 0.14 & 4.27 & 1.9 & -0.26 & 3.42 \\
\hline & 4372.43 & 3.21 & 3.0 & -0.13 & 4.00 & 1.2 & -0.53 & 3.52 \\
\hline $31 \mathrm{~F}$ & 7047.99 & 2.84 & 2.0 & 1.26 & 4.67 & - & - & - \\
\hline $34 \mathrm{~F}$ & 5477.25 & 3.32 & 2.0 & 0.18 & 3.83 & - & - & - \\
\hline & 5746.96 & 3.18 & 6.0 & 0.45 & 4.10 & 1.0 & -0.33 & 3.92 \\
\hline $35 \mathrm{~F}$ & 5163.94 & 3.37 & 8.0 & 0.40 & 4.07 & 1.0 & -0.51 & 3.22 \\
\hline & 5283.10 & 3.37 & 3.0 & 0.29 & 4.01 & - & - & - \\
\hline $39 \mathrm{~F}$ & 5551.53 & 3.89 & 3.0 & 0.68 & 3.94 & - & - & - \\
\hline $43 \mathrm{~F}$ & 6944.91 & 3.80 & 3.0 & 0.90 & 3.80 & - & - & - \\
\hline $44 \mathrm{~F}$ & 6188.55 & 3.95 & 1.0 & 0.94 & 3.87 & - & - & - \\
\hline a2G-a2I & 5870.00 & 4.10 & 2.0 & 0.37 & 3.39 & - & - & - \\
\hline & 6044.10 & 4.08 & 3.0 & 0.73 & 3.75 & - & - & - \\
\hline a2G-b2D & 4898.61 & 4.50 & 4.0 & 0.42 & 3.60 & - & - & - \\
\hline & 5060.08 & 4.74 & 3.0 & 0.60 & 3.78 & - & - & - \\
\hline a2G-c2G & 5673.22 & 4.10 & 6.0 & 0.65 & 3.67 & - & - & - \\
\hline & 5835.44 & 4.70 & 5.0 & 0.65 & 3.66 & - & - & - \\
\hline a2P-c2D & 5048.18 & 4.70 & 3.0 & 0.72 & 3.72 & - & - & - \\
\hline
\end{tabular}



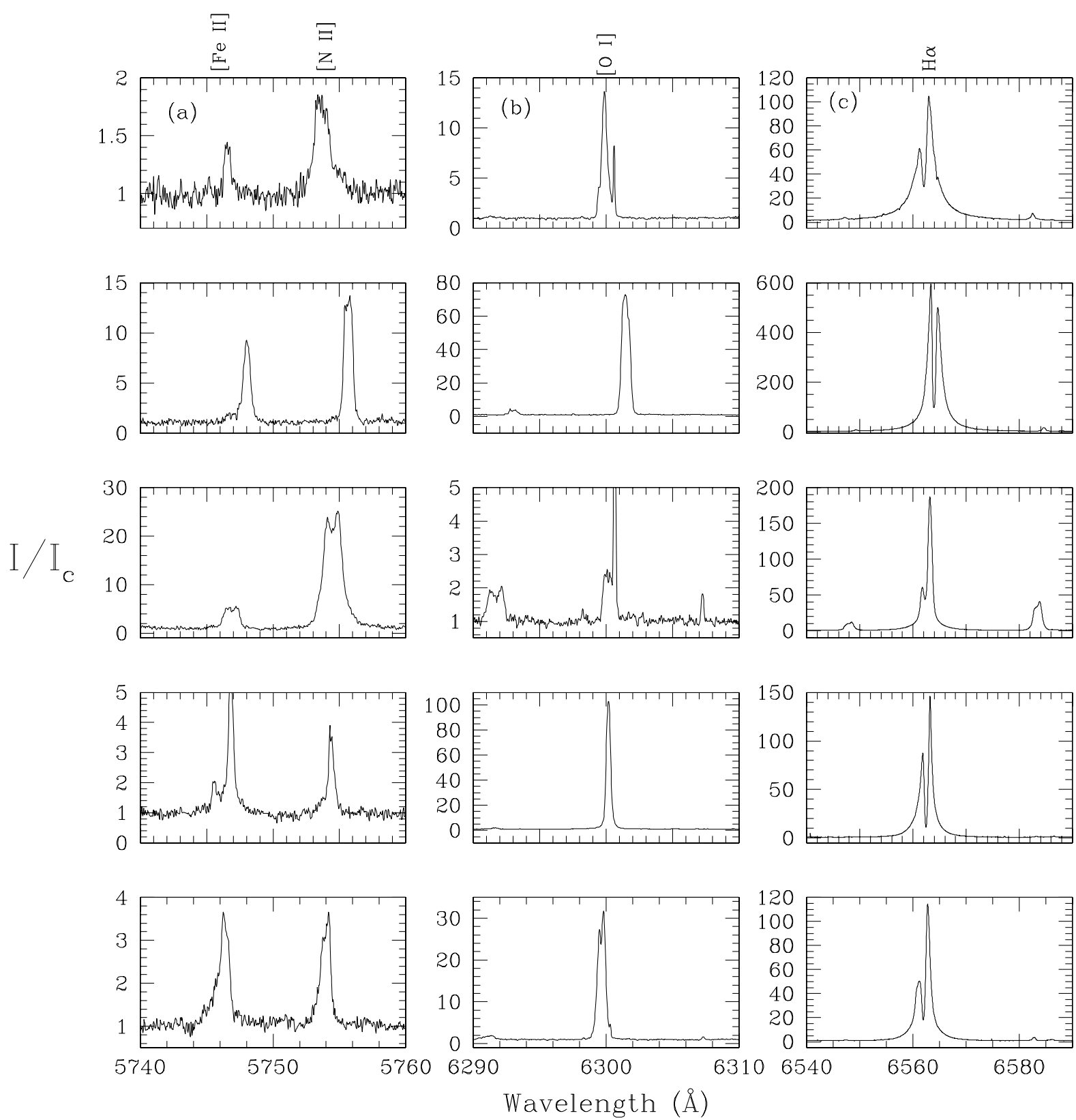

Fig. 2. High-resolution spectra obtained with FEROS spectrograph in the region around the forbidden nitrogen line at $5754 \AA$ (a)), $6300 \AA$ (b)), and $\mathrm{H} \alpha(\mathbf{c}))$. From top to bottom we show the spectra of SS73 21, RJHA 49, SS73 11, SS73 56, and Hen 2-79. Notice the strength of H $\alpha$ in RJHA 49. The intensities are in continuum units.

Parthasarathy et al. (2001) as 1.3 and 0.75 respectively. There was no previous extinction determination in the direction of RJHA 49. Probably the main reason for this difference between our derived value for SS73 21 and the one obtained by García-Lario et al. (1999) is that the $\mathrm{H} \alpha$ shows a double-peak profile. Therefore, Balmer lines tend to deviate from pure case $\mathrm{B}$ recombination; due to that, the derereddened $\mathrm{H} \alpha / \mathrm{H} \beta$ ratio clearly disagrees from case B recombination. A similar effect is seen in symbiotic stars (Oliversen \& Anderson 1982).

\section{Physical conditions}

\subsection{Excitation temperature}

Since we observe several emission lines of forbidden single ionized iron in the spectra of RJHA 49 and SS73 21, it is possible to derive the excitation temperature in the emitting region by following the description of Viotti (1969; see also Thackeray 1967). This procedure was previously used by us for SS73 11, SS73 56, and Hen 2-79.

In Table 2 we show the parameter $\beta$ (6th and 9th columns) defined as $\beta=\log \left(F_{\mathrm{c}}(\lambda) \lambda(\AA) / g A\right)$ and the excitation potential of the forbidden lines used in the calculation ( 3 rd column). In the above expression, $F_{\mathrm{c}}(\lambda)$ is the line intensity in units of $\mathrm{H} \beta=$ 100 corrected for reddening, and $\lambda$ is given in Angstroms. The obtained temperature based on this method is $T_{\text {exc }}=(8300 \pm$ $850) \mathrm{K}$ for SS73 21 and $T_{\text {exc }}=(12000 \pm 1100) \mathrm{K}$ for RJHA 49.

\subsection{Electron density}

The electron density was obtained using the dereddened [N II] $\lambda 6584 / 5754$ ratio. For RJHA 49 and SS73 21, the ratios 
Table 3. Equivalent widths and intensities relative to the continuum of some lines in the spectra of RJHA 49 and SS73 21.

\begin{tabular}{lc|cc|cc}
\hline \hline Wavelength & Identification & \multicolumn{2}{|c|}{ RJHA 49 } & \multicolumn{2}{c}{ SS7321 } \\
Line & $\lambda_{\text {lab }}$ & $I / I_{\mathrm{c}}$ & $W_{\lambda}(\AA)$ & $I / I_{\mathrm{c}}$ & $W_{\lambda}(\AA)$ \\
\hline $\mathrm{H} \gamma$-blue & 4340 & 11 & 3 & 3 & 4 \\
$\mathrm{H} \gamma$-red & & 11 & 6 & 8 & 9 \\
$\mathrm{H} \beta$-blue & 4861 & 18 & 11 & 9 & 8 \\
$\mathrm{H} \beta$-red & & 19 & 16 & 23 & 26 \\
Fe II & 4923 & 23 & 11 & 4 & 2 \\
Fe II & 5018 & 36 & 20 & 6 & 3 \\
Fe II & 5169 & 23 & 15 & 4 & 2 \\
Fe II & 5316 & 45 & 33 & 9 & 5 \\
{$[\mathrm{~N}$ II } & 5754 & 12 & 8 & 2 & 1 \\
$\mathrm{He}$ I & 5876 & - & - & 4 & 5 \\
{$[\mathrm{O}$ I] } & 6300 & 75 & 38 & 12 & 7 \\
$\mathrm{H} \alpha$-blue & 6563 & 595 & 77 & 66 & 52 \\
$\mathrm{H} \alpha$-red & & 502 & 79 & 114 & 82 \\
{$[$ Fe II] } & 7155 & 31 & 24 & 5 & 3 \\
O I & 8446 & 126 & 150 & 28 & 42 \\
$\mathrm{Ca}$ II & 8498 & 9 & 7 & 11 & 15 \\
$\mathrm{Ca}$ II & 8662 & 3 & 2 & 11 & 13 \\
\hline
\end{tabular}

are 3.0 and 8.3, respectively. Adopting an electron temperature of $10000 \mathrm{~K}$, the electron density is $8.2 \times 10^{5} \mathrm{~cm}^{-3}$ for RJHA 49 and $2.6 \times 10^{5} \mathrm{~cm}^{-3}$ for SS73 21. García-Lario et al. (1999) find a value for SS73 21 compatible to ours $\left(\sim 2 \times 10^{5} \mathrm{~cm}^{-3}\right)$.

\section{The nature of RJHA 49 and SS73 21}

Although we have investigated the spectrum of RJHA 49 in some detail and have estimated some physical conditions of its envelope, it is still difficult to determine its evolutionary status, since its distance is not well constrained, and thus its luminosity cannot be accurately derived. The lack of wide and narrow band images such as the ones made in the case of SS73 21 (Sahai et al. 1999) also complicates this kind of discussion. However, according to Zickgraf (1989), it is possible to use [N II] and $[\mathrm{O} \mathrm{I}]$ to distinguish a $\mathrm{B}[\mathrm{e}] \mathrm{sg}$ star from an LBV. By following this criteria, since the [OI] $\lambda 6300$ line is more intense than the [N II] $\lambda 5754$ (see Fig. 2), we conclude that RJHA 49 is either a $\mathrm{B}[\mathrm{e}]$-supergiant or a proto-planetary nebula.

Although the emission-line spectra of RJHA 49 and SS73 21 and the other stars look similar to $\eta$ Car (Fig. 1) this does not mean that they are in the same evolutionary stage. Hillier et al. (2001) report that the spectrum of $\eta$ Car taken at high spatial resolution $\left(0{ }^{\prime} 1 \times 00^{\prime} 13\right)$ with the Hubble Space Telescope $(H S T)$ is considerably different from the ones obtained with ground telescopes. Low-resolution spectroscopic observation of $\eta$ Car like the one presented in our Fig. 1, results in a combination of nebular (mainly from the Weigelt blobs) and central source spectra, i.e., in narrow permitted and forbidden lines (e.g. [Fe II], Fe II, and $\mathrm{HI}$ ) superimposed on a broad emission-line spectrum.

As mentioned, SS7321 was first suggested to be a proto planetary-nebula by Parthasarathy \& Pottasch (1989), according to its far-infrared IRAS colors. Recently, HST images have revealed long cylindrical-shaped bipolar lobes, surrounded by a faint elliptical halo, which is possibly a remnant of the AGB phase (Sahai et al. 1999). These characteristics were almost simultaneously observed by Gárcia-Lario et al. (1999) through images and optical and near-infrared spectroscopy. The presence of a circumstellar disk is also inferred from these studies and from polarization data (Scarrot \& Scarrot 1995).
The amount of reddening in the direction of SS73 21 can help for constraining its luminosity and thus its nature. However, different values exist for this quantity. If we consider a visual extinction of about $7 \mathrm{mag}$, as in the work of Sahai et al. (1999), we would have a luminosity of approximately $28000 L_{\odot}$ at a distance of $3 \mathrm{kpc}$ (Bujarrabal \& Bachiller 1991). This places this star in the HR diagram among Be supergiants (Fig. 8 of Miroshnichenko et al. 2001), for a probable temperature of $\sim 22000 \mathrm{~K}$ (Sahai et al. 1999). On the other hand, by considering a visual extinction of $2.4 \mathrm{mag}$ (from the present work), we arrive at a much lower value of $2000 L_{\odot}$ for the luminosity. This estimate shows better agreement with a proto-planetary nebula status for SS73 21, which has been claimed in the literature.

Our analysis of the high-resolution data of SS73 21 shows important spectral characteristics not seen in previous studies. An important matter highlighted in the literature is that the broad, non-Gaussian profile of the $\mathrm{H} \alpha$ line seen in lowresolution spectra of SS73 21 and of some other protoplanetary nebula (e.g. M 2-9) is due to mass outflows. Indeed, Parthasarathy et al. (2001) suggests that the asymmetry in $\mathrm{H} \alpha$ seen in SS73 21 is probably due to a P-Cygni profile. However, as presented in Sect. 2.2.2, our high-resolution spectrum of SS73 21 reveals an $\mathrm{H} \alpha$ with a double-peak profile. This fact is compatible with the presence of a circumstellar disk, which was deduced from the studies above mentioned.

Another very interesting characteristic revealed by the highresolution spectrum of SS73 21 is that some He I lines resemble a P-Cygni profile. This can be seen in Fig. 3 for the transitions He I $\lambda 5015, \lambda 5876$, and $\lambda 6678$. If these lines are indeed in P-Cygni, it is direct evidence that mass-loss is active in SS73 21. We found no other lines of other ions with such profiles. At present, it would be very difficult to determine the formation region of these emissions. A stellar wind of the central star could be a possibility, but P-Cygni lines can also be formed in a strong bipolar outflow. Clearly, it would be valuable to obtain a mass-loss rate estimate. However, this is beyond the scope of the present paper, since it would require the use of radiative transfer models.

An estimate for the expansion velocity involved can be made from the He I $\lambda 5015$ line. We chose not to consider the He I $\lambda 5876$ and He I $\lambda 6678$ lines shown in Fig. 3 in this calculation. For the former, the wavelength coverage of the absorption part of the profile is very uncertain. In the latter transition, the absorption is barely visible. Before computing the expansion velocity, we derived a radial velocity $\left(V_{\text {rad }}\right)$ for SS73 21 of approximately $+63 \mathrm{~km} \mathrm{~s}^{-1}$ from a comparison between the laboratory and observed wavelengths of several emission lines. After correcting for $V_{\text {rad }}$ and considering the wavelength where the absorption part of the He I $\lambda 5015$ profile returns to the continuum, we finally derived a value of $\sim 90 \mathrm{~km} \mathrm{~s}^{-1}$. This value is lower than the terminal velocities usually found in central stars of planetary nebulae, which reach hundreds or even thousands of $\mathrm{km} \mathrm{s}^{-1}$ (e.g. Hultzsch et al. 2007), and it is considerably higher than the expansion velocity related to molecular transitions of $\mathrm{CO}$ in SS73 21, which is $\sim 15 \mathrm{~km} \mathrm{~s}^{-1}$ (Bujarrabal \& Bachiller 1991).

\section{Summary and conclusions}

We have analyzed low and high resolution optical spectra of two peculiar emission-line stars: RJHA 49 and SS73 21. We performed line measurements and identifications for several features in their spectra. The spectrum of RJHA 49 has been analyzed in detail for the first time. For both objects, a set of iron 


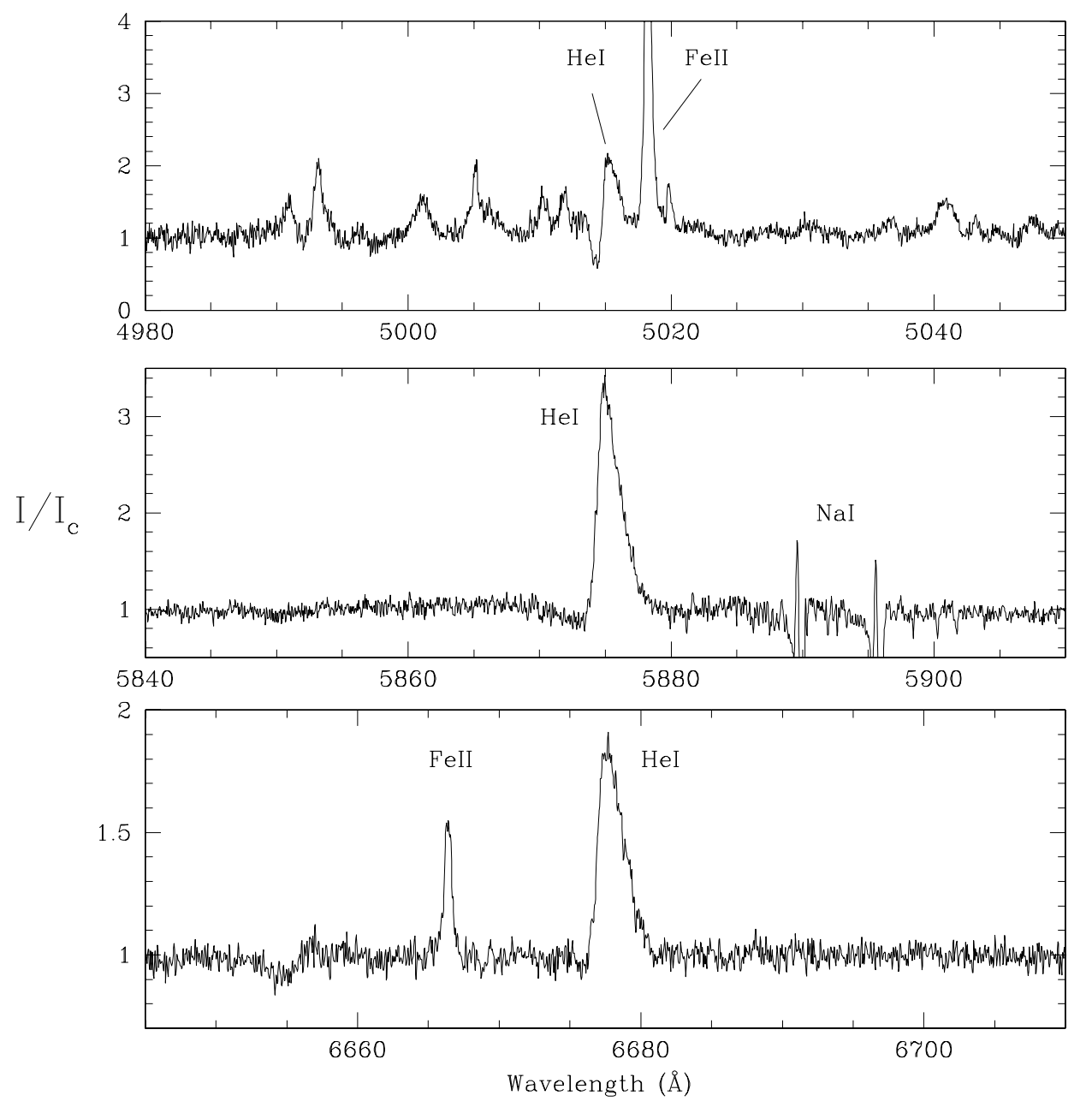

Fig. 3. Helium lines in SS73 21 with apparent P-Cygni profiles. The intensities are in continuum units.

and nitrogen forbidden lines were used to derive the interstellar reddening, the excitation temperature, and the electron density. We showed that the $\mathrm{H} \alpha$ line in SS73 21 presents a doublepeak profile, in contrast the suggestion in the literature that it should reveal a P-Cygni with high resolution data. The presence of the double peak implies that the determination of $E(B-V)$ from the $\mathrm{H} \alpha / \mathrm{H} \beta$ ratio is not reliable, since we cannot securely assume case B recombination. We found that some He I lines in SS73 21 resemble a P-Cygni profile, directly suggesting that mass loss is active in this star. Finally, we discussed the nature of both objects, where the status of SS7321 is clearer (a proto-planetary nebula) than for RJHA 49. Solely from the [O I] and [N II] line strengths, RJHA 49 is either a B[e]-supergiant or a proto-planetary nebula. In order to gain more insight into the evolutionary stages of these and other similar peculiar emission line stars, distance estimates, high angular resolution spectroscopy, as well as wide and narrow band images would be very desirable.

Acknowledgements. W.M. acknowledges the CNPq for financial support (postdoc position - 151635).

\section{References}

Allen, D. A., \& Swings, J. P. 1976, A\&A, 47, 293

Bujarrabal, V., \& Bachiller, R. 1991, A\&A, 242, 247
García-Lario, P., Riera, A., \& Manchado, A. 1999, ApJ, 526, 854 Hamuy, M., Suntzeff, N. B., Heathcote, S. R., et al. 1994, PASP, 106, 566 Hillier, D. J., Davidson, K., Ishibashi, K., \& Gull, T. 2001, ApJ, 553, 837 Hultzsch, P. J. N., Puls, J., Méndez, R. H., et al. 2007, A\&A, 467, 1253 Jaschek, M., Andrilatt, Y., \& Jaschek, C. 1996, A\&AS, 120, 99

Kaufer, A., Stahl, S., Tubbesing, S., et al. 1999, The Messenger, 95, 8 Kohoutek, L. 1971, A\&A, 13, 493

Lamers, H. J. G. L. M., Zickgraf, F. J., de Winter, D., Houziaux, L., \& Zorec, J. 1998, A\&A, 240, 117 (L98)

Landaberry, S. J. C., Pereira, C. B., \& de Araújo, F. X. 2001, A\&A, 376, 917 (L01)

Miroshnichenko, A. S. 2006, ASP Conf. Ser., 355, 13

Miroshnichenko, A. S., Levato, H., Bjorkman, K. S., \& Grosso, M. 2001, A\&A, 371,600

Oke, J. B. 1974, ApJS, 27, 21

Oliversen, N. A., \& Anderson, C. M. 1982, in The Nature of Symbiotic Stars, ed. M. Friedjung, \& R. Viotti (Dordrecht: Reidel), IAU Coll., 70, 71

Pagel, B. E. J. 1969, Nature, 221, 325

Parthasarathy, M., \& Pottasch, S. R. 1989, A\&A, 225, 521

Parthasarathy, M., García-Lario, P., Gauba, G., et al. 2001, A\&A, 376, 941

Pereira, C. B., Franco, C. S., \& de Araújo, F. X. 2003a, A\&A, 397, 927

Pereira, C. B., Landaberry, S. J. C., \& de Araújo, F. X. 2003b, A\&A, 402, 693

Sahai, R., Bujarrabal, V., \& Zijlstra, A. 1999, ApJ, 518, L115

Sanduleak, N., \& Stephenson, C. B. 1973, ApJ, 185, 899

Scarrot, S. M., \& Scarrot, R. M. J. 1995, MNRAS, 277, 277

Swings, J. P., \& Allen, D. A. 1973, ApL, 14, 65

Thackeray, A. D. 1967, MNRAS, 135, 51

The, P. S., de Winter, D., \& Pérez, M. R. 1994, A\&AS, 104, 315

Viotti, R. 1969, Ap\&SS, 5, 323

Zickgraf, F. J. 1989, in Angular Momentum and Mass Loss for Hot Stars, ed. R. A. Wilson, \& R. Stalio (Kluwer Academic Publishers), 245 